\title{
In vitro activity of azithromycin in Salmonella isolates from Pakistan
}

\author{
Farhana Butt ${ }^{1}$ and Faisal Sultan ${ }^{2}$ \\ Department of Pathology ${ }^{1}$ and Department of Medicine ${ }^{2}$, Shaukat Khanum Memorial Cancer Hospital and Research \\ Center, Lahore, Pakistan
}

\begin{abstract}
Introduction: Enteric fever is caused by Salmonella enterica serovars Typhi and Paratyphi A, B and C. It is a significant public health issue in Pakistan, which is exacerbated by a high level of resistance some isolates display to drugs routinely used in treatment. Azithromycin may be a treatment option for such isolates.

Methodology: We determined the minimum inhibitory concentrations (MICs) of Salmonella Typhi and Paratyphi isolates against azithromycin in an attempt to gauge its feasibility as a therapeutic option. The MICs were also compared with corresponding disc diffusion zone sizes to see if there was consistency between the two tests. We tested 45 Salmonella enterica isolates using E-tests for MIC detection and azithromycin discs with a concentration of $15 \mu \mathrm{g} / \mathrm{ml}$ for disc diffusion testing.

Results: Salmonella Typhi, Salmonella Paratyphi A, and Salmonella Paratyphi C isolates demonstrated MICs of 2-12mg/L against azithromycin, suggesting that the antibiotic could be used for therapeutic purposes. For Salmonella Paratyphi B, the MICs were 2-48 mg/L. The higher MIC indicates a need for caution when considering use of azithromycin for Salmonella Paratyphi B infections without first testing for the MIC. There was a close correlation between MICs and zone sizes which was statistically significant.

Conclusions: Our results indicate azithromycin is a potential therapeutic option for enteric fever. Standardized laboratory testing methods and interpretation for azithromycin against Salmonella enterica would allow laboratories to report upon this antibiotic with confidence.
\end{abstract}

Key words: Salmonella enterica; typhoid; enteric fever; azithromycin; resistance; Pakistan

J Infect Dev Ctries 2011; 5(5):391-395.

(Received 23 February 2010 - Accepted 06 October 2010)

Copyright $(\mathcal{C} 2011$ Butt and Sultan. This is an open-access article distributed under the Creative Commons Attribution License, which permits unrestricted use, distribution, and reproduction in any medium, provided the original work is properly cited.

\section{Introduction}

Enteric fever is caused by Salmonella enterica serovars Typhi and Paratyphi A, B and C. The World Health Organization has calculated the crude incidence of typhoid fever alone (caused by Salmonella Typhi) for South East Asia to be $110 / 100,000$ persons per year [1]. Studies from Pakistan indicate the incidence here may be even higher [2,3]. In addition to the high incidence of disease, Pakistan also has a high incidence of reduced quinolone susceptibility, with isolates of minimum inhibitory concentration (MIC) $\geq 1 \mathrm{mg} / \mathrm{L}$ accounting for up to $64 \%$ Salmonella Typhi (S. Typhi) [3]. Within our own hospital, of the 85 Salmonella enterica isolates cultured between 2007 and 2008, $79 \%$ were resistant to nalidixic acid, a good indicator of poor response to ciprofloxacin (unpublished data). Multi-drug resistance which includes resistance to ampicillin, chloramphenicol and co-trimoxazole is also high in Pakistan (45\% for $S$. Typhi) [3].

Treatment options for multi-drug resistant (MDR) and quinolone-resistant isolates include parenteral ceftriaxone, oral cefixime, and oral azithromycin [4].

Increased use of cephalosporins for treatment of typhoid fever by clinicians familiar with them may be contributing to increased resistance to thirdgeneration cephalosporins $[4,5,6]$. Azithromycin is an attractive alternative to the cephalosporins used (parenteral ceftriaxone and oral cefixime) in view of its single daily dosing, possibility of use in $\beta$-lactam allergic patients, and lower cost.

Several studies have documented the efficacy of azithromycin in the treatment of uncomplicated enteric fever. However, these results have been based mainly upon clinical criteria without correlating success to specific laboratory-based breakpoints for isolate susceptibility and resistance. Possible reasons for this situation could be the lack of interpretive guidelines for assessing Salmonella species' susceptibility towards azithromycin, and the pharmacodynamics of the drug.

For the above-mentioned reasons, this study was designed to determine MICs of azithromycin against 
Table 1. MICs of 45 Salmonella enterica isolates.

\begin{tabular}{|c|c|c|c|c|c|}
\hline \multirow{2}{*}{$\begin{array}{l}\text { Azithromycin } \\
\text { MIC value } \\
\mathrm{mg} / \mathrm{L}\end{array}$} & \multirow{2}{*}{$\begin{array}{l}\text { Zone diameters } \\
(\mathrm{mm}) \text { observed for } \\
\text { corresponding MIC }\end{array}$} & \multicolumn{4}{|c|}{ Number of isolates for each MIC value } \\
\hline & & $\begin{array}{l}\text { S. Typhi } \\
\text { (Total 22) }\end{array}$ & $\begin{array}{l}\text { S.Paratyphi A } \\
\text { (Total 17) }\end{array}$ & $\begin{array}{l}\text { S. Paratyphi B } \\
\text { (Total 5) }\end{array}$ & $\begin{array}{l}\text { S. Paratyphi C } \\
\text { (Total 1) }\end{array}$ \\
\hline 2 & $18-23$ & 7 & 4 & 2 & 0 \\
\hline 4 & $16-20$ & 9 & 5 & 1 & 0 \\
\hline 8 & $14-17$ & 5 & 5 & 0 & 1 \\
\hline 16 & $10-16$ & 1 & 3 & 1 & 0 \\
\hline 48 & 6 & 0 & 0 & 1 & 0 \\
\hline
\end{tabular}

Salmonella enterica isolates. To the best of our knowledge, such MICs have not been reported in isolates from Pakistan. Our aim was to obtain baseline data which could be built upon in the future for correlation with clinical outcomes. We also compared MICs with corresponding disc diffusion zone sizes to see if there was consistency between the two tests.

\section{Methodology}

From samples received at Shaukat Khanum Memorial Cancer Hospital laboratory between November 2007 and June 2009, a collection of 45 Salmonella enterica isolates were tested. Blood cultures were the commonest source (38) and the isolates included $22 S$. Typhi, $17 S$. Paratyphi A, $5 S$. Paratyphi B and one $S$. Paratyphi C.

The organisms were identified using phenotypic colony characteristics and confirmed with API 20E (bioMerieux SA, Marcy I'Etoile, France) and serotyping (Antiserum Salmonella Polyvalent BioRad, Marnes-la-Coquette, France). Disc diffusion testing was conducted according to the Kirby-Bauer method using a bacterial suspension of 0.5 McFarland turbidity to inoculate the surface of a Mueller-Hinton agar plate (Oxoid, Hampshire, UK). Fifteen $\mu \mathrm{g} / \mathrm{ml}$ azithromycin discs (Oxoid, Hampshire, UK) were used and plates were then incubated for 18 to 20 hours at ambient air conditions. MICs were determined using E-test strips (AB Biodisk, Solna, Sweden) which were set up simultaneously with the disc diffusion test, using the same 0.5 McFarland organism suspension with Mueller-Hinton agar (Oxoid, Hampshire, UK) and incubated under the same conditions. Staphylococcus aureus ATCC 25923 and ATCC 29213 were used as controls for the disc diffusion and MIC testing respectively.

To test the association between the two variables under study, which were zone diameter in $\mathrm{mm}$ and MIC in $\mathrm{mg} / \mathrm{L}$, the Statistical Package for Social
Sciences (SPSS) version 10 (IBM, Chicago, Illinois, USA) was used.

\section{Results and discussion}

While there are no official breakpoints for azithromycin susceptibility and resistance given against $S$ Typhi and $S$ Paratyphi, a few studies have attempted to correlate successful therapy with the MICs of infecting isolates. In these studies MICs tend not to exceed $32 \mathrm{mg} / \mathrm{L}[7,8,9]$. The British Society for Antimicrobial Chemotherapy (BSAC) also mentions azithromycin being used in treatment of infections with isolate MICs of less than $16 \mathrm{mg} / \mathrm{L}$ [10], but falls short of providing any specific guidance for testing.

Within this context, while our sample size was small, we can comment on two observations based on our findings detailed in Table 1 . For $S$. Typhi, $S$. Paratyphi A and $S$. Paratyphi C, azithromycin MICs were observed between $2-12 \mathrm{mg} / \mathrm{L}$ suggesting that azithromycin could be used for therapeutic purposes where indicated. However, $S$. Paratyphi B displayed a tendency towards higher MICs and possible resistance.

Nevertheless, as mentioned above, most studies use clinical criteria to gauge therapeutic success, referring to the laboratory only to confirm clearance of the organism from blood or urine and without necessarily correlating this to laboratory MICs. This use of clinical criteria alone could be due to a lack of definitive laboratory guidance. Another contributing factor could be the pharmacodynamics of azithromycin whereby clinical success is reported despite peak serum levels of $0.4 \mathrm{mg} / \mathrm{mL}$ following a $500 \mathrm{mg}$ oral dose [9, 11], which is far less than laboratory reported MICs. The reason for therapeutic response is the high intracellular concentrations achieved by azithromycin of up to 50 to 100 times that in serum $[9,12]$.

Given these factors, two questions may be posed: (1) Should laboratories be concerned with Salmonella 
Figure 1. Scatterplot of azithromycin MIC $(\mathrm{mg} / \mathrm{L})$ and disc zone size $(\mathrm{mm})(\mathrm{N}=45)$

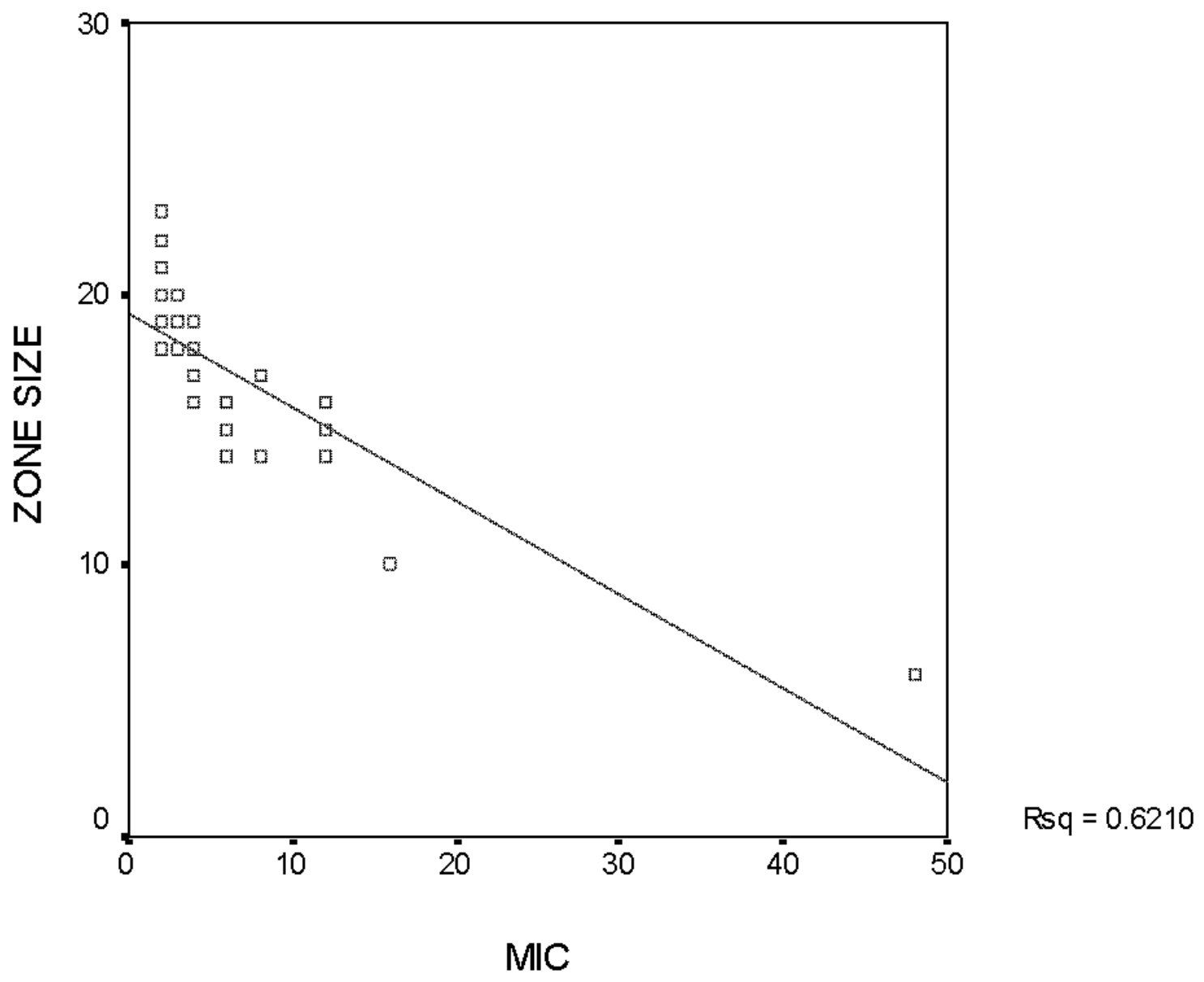

testing against azithromycin? and (2) is it truly feasible to continue treatment without referring to laboratory susceptibility results? When answering these questions, the following must be taken into consideration. While $S$. enterica serovar Typhi occupies a predominantly intracellular location, it is estimated that one-third of bacterial cells in the blood are extracellular [13]. Exposure of such isolates to sub-optimal levels of azithromycin can lead to treatment failure and development of resistance [9]. While it is more likely azithromycin would be used in treatment of MDR-strains, these strains are also more likely to have a higher extra-cellular concentration of organisms [13]. Also of interest is the recent report of azithromycin treatment failure following its use in a shigellosis outbreak in Paris, linked to plasmidmediated resistance to macrolides [14]. It is quite possible that such resistance could be transmitted to Salmonella spp. and the above scenario could facilitate this occurrence.
From this perspective, it would seem that if we are to ensure the continued development and progression of azithromycin as a viable treatment option for enteric fever, laboratory testing may have a significant role to play. To do this, a number of variables associated with laboratory testing need to be addressed. Methods used to test Salmonella against azithromycin are not always described in detail and specifics about techniques, media and $\mathrm{pH}$ cannot always be ascertained or easily replicated. Reproducibility is of relevance as studies have shown variation in MICs related to media $\mathrm{pH}$ and inoculum size [15]. There may also be some differences between results with E-test strips and agar dilution methods [16].

Therefore, ensuring the uniformity of methods employed when testing would be useful. Using disc diffusion and E-test strips for testing, materials and methods readily available in a general microbiology laboratory, we observed a close correlation between MICs and zone size. The Pearson's correlation 
between the two variables was found to be significant $(-0.79$, p-value $<0.001)$ and showed a negative correlation between the zone size and MIC. Also, a simple linear regression was used to test whether the zone size can predict MIC. The test was significant at an alpha-level of $0.05(\mathrm{~F}=70.4, \mathrm{p}<0.001)$. $\mathrm{R}$ squared $=0.62$; therefore, $62 \%$ of the variance in MIC can be explained by differences in zone sizes.

While relatively basic, our methods are readily reproducible in a routine laboratory. Subsequent submission of isolates to more specialized facilities can follow to allow the development of more sophisticated interpretation and generalization of the findings.

\section{Conclusion}

Increasing cephalosporin resistance in Salmonella enterica isolates necessitates the availability of alternative therapies for enteric fever in countries with a high disease burden. Our study results suggest azithromycin may be one of the therapeutic options. However, we run the risk of exposing patients with MDR strains and significant extracellular bacteremia to sub-optimal therapy. Progression to azithromycin resistance may occur before clinicians start really utilizing it. Recommendations for azithromycin testing against Salmonella enterica would facilitate laboratories in reporting upon this antibiotic with confidence and allow a more accurate susceptibility pattern to emerge.

\section{Acknowledgements}

We would like to acknowledge Ms. Zunaira Rao for her assistance in conducting the laboratory work and Dr. Farhana Badar for conducting the statistical analysis.

\section{References}

1. Crump JA, Luby SP, Mintz ED (2004) The global burden of typhoid fever. Bull World Health Organ 82: 346-353.

2. Siddiqui FJ, Rabbani F, Hasan R, Nizami SQ, Bhutta ZA (2006) Typhoid fever in children: some epidemiological considerations from Karachi Pakistan. Int J Infect Dis 10: 215-222.

3. Hasan R, Zafar A, Abbas Z, Mahraj V, Malik F, Zaidi A (2008) Antibiotic resistance among Salmonella enterica serovars Typhi and Paratyphi A in Pakistan (2001-2006). J Infect Developing Countries 2: 289294.

4. World Health Organization Department of Vaccines and Biologicals. Background document: the diagnosis, prevention and treatment of typhoid fever. Geneva: WHO 2003:

19-23. (www.who.int/entity/vaccine_research/documents/en/t yphoid_diagnosis.pdf).

5. Capoor MR, Nair D, Hasan AS, Aggarwal P, Gupta B (2006) Narrowing therapeutic options in typhoid fever, India. Southeast Asian J Trop Med Public Health 37: 1170-1174.

6. Pokharel BM, Koirala J, Dahal RK, Mishra SK, Khadga PK, Tuladhar NR (2006) Multidrug-resistant and extended-spectrum $\beta$-lactamase (ESBL)producing Salmonella enterica (serotypes Typhi and Paratyphi A) from blood isolates in Nepal: surveillance of resistance and a search for newer alternatives. Int J Infect Dis 10: 434-438.

7. Girgis NI, Bulter T, Frenck RW, Sultan Y, Brown FM, Tribble D, Khakhria R (1999) Azithromycin versus ciprofloxacin for treatment of Uncomplicated Typhoid fever in a randomized trial in Egypt that included patients with Multidrug Resistance. Antimicrob Agents Chemother 43: 1441-1444.

8. Chinh NT, Parry CM, Ly NT, Ha HD, Thong MX, Deip TS, Wain J, White NJ, Farrar JJ (2000) A randomized controlled comparison of azithromycin and Ofloxacin for treatment of multidrug-resistant or Nalidixic acid - resistant enteric fever. Antimicrob Agents Chemother 44: 1855-1859.

9. Parry CM, Ho VA, Phuong LT, Bay PVB, Lanh MN, Tung LT, Tham NTH, Wain J, Hien TT, Farrar J (2007) Randomized controlled comparison of Ofloxacin, Azithromycin and an OfloxacinAzithromycin combination for treatment of Multidrug-resistant and Nalidixic Acid-resistant Typhoid Fever. Antimicrob Agents Chemother 51: 819-825.

10. Andrews JM, BSAC Working Party on Susceptibility Testing (2008) BSAC standardized disc susceptibility testing method (version 7). J Antimicrob Chemother 62: 256-278.

11. Foulds G, Shepard RM, Johnson RB (1990) The pharmacokinetics of azithromycin in human serum and tissues. J Antimicrob Chemother 25 Suppl A: 7382.

12. Butler T and Girard AE (1993) Comparative efficacies of azithromycin and ciprofloxacin against experimental Salmonella typhimurium infection in mice. J Antimicrob Chemother 31: 313-319.

13. Wain J, Diep TS, Ho VA, Walsh AM, Hoa NTT, Parry CM, White NJ (1998) Quantitation of bacteria in blood of typhoid fever patients and relationship between counts and clinical features, transmissibility, and antibiotic resistance. J Clin Microbiol 36: 16831687.

14. Nguyen MCP, Woerther P-L, Bouvet M, Andremont A, Leclercq R, Canu A (2009) Escherichia coli as Reservoir for Macrolide Resistance Genes. Emerg Infect Dis 15: 1648-1650.

15. Butler T, Frenck RW, Johnson RB, Khakhria R (2001) In vitro effects of azithromycin on Salmonella Typhi: 
early inhibition by concentrations less than the MIC and reduction of MIC by alkaline $\mathrm{pH}$ and small inocula. J Antimicrob Chemother 47: 455-458.

16. Chayani N, Tiwari S, Sarangi G, Mallick B, Mohapatra A, Paty BP, Das P (2009) Role of azithromycin against clinical isolates of family enterobacteriaceae: A comparison of its minimum inhibitory concentration by three different methods. Indian J Med Microbiol 27: 107-110.

\section{Corresponding author}

Farhana Butt

19 Livingstone Road

Hounslow, Middlesex TW3 1XX

United Kingdom

Telephone: +44 (0)208 5704712; $\quad$ Mobile +44 (0)7707339503

Email: microreg@msn.com

Conflict of interests: No conflict of interests is declared. 